\title{
Imposing strong correlated energy spread on relativistic bunches with transverse deflecting cavities
}

\author{
Nikolai Yampolsky®* and Evgenya I. Simakov® \\ Los Alamos National Laboratory, Los Alamos, New Mexico 87545, USA \\ Alexander Malyzhenkov ${ }^{\dagger}$ \\ Los Alamos National Laboratory, Los Alamos, New Mexico 87545, USA \\ and Northern Illinois Center for Accelerator and Detector Development and Department of Physics, \\ Northern Illinois University, DeKalb, Illinois, USA
}

(Received 10 October 2019; accepted 13 April 2020; published 14 May 2020)

\begin{abstract}
We demonstrate a novel scheme for imposing or removing large energy chirp on relativistic electron bunches. This method relies on the transverse-to-longitudinal mixing accomplished with a set of transverse deflecting cavities. The working principles of the new concept are explained using the first-order matrix formalism in the approximation of the linear single-particle dynamics. The scheme demonstrates versatility regarding the average beam energy and, hence, positioning along the accelerator beam line. The performance of the scheme is numerically investigated with the ELEGANT particle-tracking code. It is shown that the associated nonlinear effects causing emittance deterioration for the extreme quantities of the total energy spread can be effectively compensated by optimization of the Twiss parameters while relying on the eigenemittance analysis. The impact of the longitudinal space charge effects on the beam dynamics in the proposed scheme is also investigated.
\end{abstract}

DOI: 10.1103/PhysRevAccelBeams.23.054403

\section{INTRODUCTION}

Relativistic electron accelerators are used for highenergy physics studies [1-3] and as light sources [4-12]. These applications require high quality and large peak current electron bunches. Such beams are subject to coherent synchrotron radiation (CSR) [13-16], which results in the variation of the beam energy along the bunch and degradation of the beam quality. As a result, the applications demanding the highest beam quality rely on the linear accelerators $[8-10,12]$ which have a smaller number of bending magnets compared to circular accelerators [3].

The design of these accelerators includes several bunch compressors (BCs) at different energies for achieving bunches with a large peak current. Compression is achieved through imposing an energy slew along the bunch and passing it through the dispersive element such as a chicane consisting of four magnetic bends [15]. Electrons in the bunch have energy chirp. The energy of electrons is larger

\footnotetext{
*nyampols@lanl.gov

Alexander.malyzhenkov@psi.ch
}

Published by the American Physical Society under the terms of the Creative Commons Attribution 4.0 International license. Further distribution of this work must maintain attribution to the author(s) and the published article's title, journal citation, and DOI. at the tail of the bunch and smaller at the head of the bunch. Electrons with higher energy travel closer to the axis in magnetic bends due to a higher relativistic $\gamma$ factor, and their time of travel through the chicane is smaller compared to that of the low-energy electrons. As a result, higherenergy electrons at the tail of the bunch catch up with electrons at the head, and the chirped bunch compresses in time.

Conventionally, the bunches are chirped while being accelerated off crest in the radio frequency (rf) field. For the off-crest acceleration, longitudinal variation of the rf fields results in a difference in accelerating gradients along the bunch. However, the accelerating gradient for off-crest acceleration is lower than on crest. Imposing the correlated energy spread on the beam in the off-crest acceleration results in a trade-off between the accelerating gradient $\left(\sim \cos \phi_{0}\right)$ and the induced energy chirp which is defined by the $R_{65} \sim \sin \phi_{0}$ element of the linear transform matrix $R$ :

$$
R_{65}=-\frac{e E_{0} k \sin \phi_{0}}{\gamma m c^{2}} s
$$

where $E_{0}$ and $k$ are, respectively, the amplitude and wave number of the rf field, $\phi_{0}$ is the $\mathrm{rf}$ phase, $\gamma$ is the normalized average energy of the bunch, $s$ is the effective length of the rf structure, $m$ and $e$ are the mass and charge, respectively, of the electron, and $c$ is the speed of light. The larger energy chirp in the beam requires off-crest acceleration at a lower 
gradient and results in a lower final beam energy in a given linac section. For example, the off-crest acceleration results in the loss of $1.575 \mathrm{GeV}$ of the beam energy at Linac Coherent Light Source [17] [ $-43^{\circ}$ rf phase between the first bunch compressor (BC1) at $250 \mathrm{MeV}$ and the second bunch compressor (BC2) at $4.54 \mathrm{GeV}]$ and $2.2 \mathrm{GeV}$ of the beam energy at the Matter-Radiation Interactions in Extremes (MaRIE) linac [18] $\left(-20^{\circ} \mathrm{rf}\right.$ phase between $\mathrm{BC} 1$ at $250 \mathrm{MeV}$ and $\mathrm{BC} 2$ at $1 \mathrm{GeV}$ ). Removing residual chirp after the bunch compression is even more challenging, since the difference between the accelerating fields at the head and the tail of the bunch is proportional to the bunch length, $\sigma_{\Delta \gamma / \gamma}=R_{65} \sigma_{z}$. As a result, off-crest acceleration is never used for bunch dechirping in modern linacs.

In this paper, we propose an alternative method for chirping relativistic electron bunches employing transverse-to-longitudinal mixing accomplished by a set of transverse deflecting cavities (TCAVs). Such cavities operate in the $\mathrm{TM}_{110}$ deflecting mode. Both electric and magnetic fields are zero on axis at zero rf phase, and the motion of the reference particle characterizing the average beam dynamics is not affected by TCAVs. The magnetic field in a TCAV varies longitudinally, providing angular deflection to electrons which depends on their longitudinal position in the bunch. The transverse variation of the longitudinal electric field provides different accelerating gradients at different transverse locations within the bunch and may be utilized for imposing correlated energy variations within the bunch. The efficient longitudinal chirping of the bunch would require a strong correlation between the longitudinal and transverse positions of particles in the bunch. In this case, the transverse variation of the electrons' energy also manifests as a longitudinal chirp. The required $x-z$ correlation inside the bunch can be created using another TCAV, which deflects electrons at some angle proportional to their longitudinal coordinate, followed by the vacuum drift space which translates angular divergence into the transverse displacement. Similar methods of imposing $x-z$ correlation are routinely used for longitudinal diagnostics of relativistic beams [19].

The proposed transverse-deflecting-cavity-based chirper (TCBC) exploits a great flexibility in manipulations with the transverse bunch size as opposed to its length, which is fixed in relativistic beams under acceleration. As a result, the difference in accelerating fields between two ends of the bunch is determined by its transverse size, which can easily be made much larger than its length.

\section{LINEAR ANALYSIS}

In this section, we introduce a TCBC beam line consisting of three TCAVs. We analyze this scheme in the approximation of the linear single-particle dynamics and demonstrate that it is capable of imposing large longitudinal energy chirp on relativistic bunches.

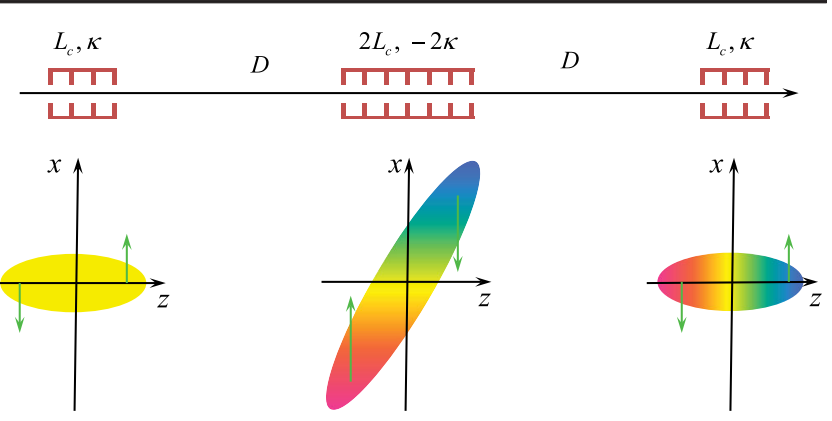

FIG. 1. The schematics of the TCBC beam line (above) consisting of three TCAVs for imposing longitudinal energy chirp in relativistic bunches. Subplots below show the $x-z$ electron bunch distribution inside each TCAV. Green arrows in these plots show $z$-dependent deflection provided by each cavity, and the color shows the particle energy at the corresponding location (larger energy is shown as red and lower energy as blue).

The schematics of the beam line is shown in Fig. 1 and consists of three cavities, namely, TCAV1, TCAV2, and TCAV3, separated by equal vacuum drifts of length $D$. The side cavities are identical, their lengths are $L_{c}$, and the normalized deflecting potential (also known as the "cavity strength") is $\kappa=e V_{\perp} /\left(m c^{2} \gamma a\right)$, where $a$ is the characteristic cavity size on the order of the rf wavelength and $V_{\perp}$ is the TCAV's deflecting voltage. The middle cavity has twice the deflecting voltage compared to the side cavities and is twice as long, assuming the same field amplitude in all TCAVs [20]. Moreover, the middle cavity has a rf phase shift of $\pi$ compared to the side cavities at the time of the bunch arrival which manifests as the deflecting voltage of an opposite sign.

Beam dynamics of the bunch along the TCBC beam line can be explained as follows. The bunch acquires angular deflection in the first cavity that varies with the $z$ coordinate and starts expanding in the drift space after the cavity. That expansion results in a strong $x-z$ correlation inside the bunch, and its transverse size becomes much larger than its length. The middle cavity changes electrons' energy based on their transverse position, which manifests as longitudinal energy chirp in a bunch with strong $x-z$ correlation. At the same time, the middle cavity produces a $z$-dependent angular kick to electrons which is twice as strong as that of the TCAV1 and has the opposite sign. As a result, the bunch is focused back in the downstream drift and restores its original shape when it arrives to the last cavity. TCAV3 predominantly compensates the residual $z$-dependent angular deflection in the beam. The beam line introduces strong correlations between the longitudinal and transverse phase spaces which are used to impose the longitudinal energy chirp on the beam with the transversely dependent accelerating field. All introduced correlations are removed at the end of the beam line, and the entire impact of the TCBC represents solely the longitudinal energy chirp, mainly imposed in the middle cavity. 
We analyze the performance of the proposed TCBC scheme within the limits of linear beam optics formalism under the assumption of the quasimonoenergetic bunch with a small angular divergence with a geometric size much smaller than the rf wavelength. The beam is represented as an ensemble of electrons characterized by coordinates in the phase space. The effect of each element of the beam line on the electron phase space is described by the beam transform matrix $M$. Our analysis is limited to the fourdimensional (4D) phase space $\left(x, x^{\prime}, z, \Delta \gamma / \gamma\right)$, since the dynamics in the transverse phase space $\left(y, y^{\prime}\right)$ is decoupled from other dimensions and manifests as a simple drift. This representation is simpler compared to the full 6D description using the full beam transport matrix $R$. The transform matrices of the drift space $M_{d}$ and the TCAV $M_{c}$ [21] are, respectively,

$$
\begin{gathered}
M_{d}(D)=\left(\begin{array}{cccc}
1 & D & 0 & 0 \\
0 & 1 & 0 & 0 \\
0 & 0 & 1 & 0 \\
0 & 0 & 0 & 1
\end{array}\right), \\
M_{c}\left(\kappa, L_{c}\right)=\left(\begin{array}{cccc}
1 & L_{c} & \frac{\kappa L_{c}}{2} & 0 \\
0 & 1 & \kappa & 0 \\
0 & 0 & 1 & 0 \\
\kappa & \frac{\kappa L_{c}}{2} & \frac{\kappa^{2} L_{c}}{6} & 1
\end{array}\right) .
\end{gathered}
$$

The overall transform matrix of the entire beam line can be found as the product of its structural components $M_{T C B C}=$ $M_{c}\left(\kappa, L_{c}\right) M_{d}(D) M_{c}\left(-2 \kappa, 2 L_{c}\right) M_{d}(D) M_{c}\left(\kappa, L_{c}\right)$. The resulting matrix is

$$
M_{T C B C}=\left(\begin{array}{cccc}
1 & 2 D+4 L_{c} & 0 & 0 \\
0 & 1 & 0 & 0 \\
0 & 0 & 1 & 0 \\
0 & 0 & -\frac{2}{3} \kappa^{2}\left(3 D+2 L_{c}\right) & 1
\end{array}\right) .
$$

The transverse and the longitudinal phase spaces are decoupled at the end of the beam line, and the start-toend transform matrix is block diagonal. The dynamics in the transverse phase space represents the drift corresponding to the overall beam line's length. The matrix element $M_{43} \equiv R_{65}<0$ results in the compressing chirp in the longitudinal phase space, since the head of the bunch $(z>0)$ loses energy $(\Delta \gamma<0)$ while the back of the bunch $(z<0)$ gains energy $(\Delta \gamma>0)$.

Equation (4) shows clear advantages of the proposed TCBC scheme over conventional off-crest acceleration. The $R_{65}$ element scales quadratically with the cavity's strength $\kappa$ as opposed to the case of the off-crest acceleration, which scales linearly with $\kappa$, yet the sign of the chirp in this novel scheme cannot be simply changed by delaying the rf phase by $180^{\circ}$. For the dipole mode, this phase delay is equivalent to the rotation of the cavity by 180。 in the $(x, y)$ plane; see Sec. IV. The quadratic scaling indicates that the TCBC is a cost-efficient solution for imposing large energy chirps. Furthermore, the imposed chirp in the TCBC scheme scales linearly with the lengths of the drifts between TCAVs. This reduces the cost of the system by replacing expensive rf cavity sections with cheap vacuum drifts.

In the limit of a large vacuum drift compared to the length of TCAVs, the chirp imposed by the TCBC can be approximated as $R_{65} \approx-2 \kappa^{2} D$. The correlated energy variation in the bunch is acquired predominantly in the middle deflecting cavity, in which the transverse beam size is the largest due to the strong transverse-to-longitudinal correlations. The transverse beam size in TCAV2 is on the order of $\sigma_{x, T C A V 2} \approx(\kappa D) \sigma_{z}$, and the chirp imposed by the TCBC beam line is on the order of $R_{65} \approx-2 \kappa\left(\sigma_{x, T C A V 2} / \sigma_{z}\right)$. Consequently, the efficiency of the TCBC over the conventional off-crest acceleration in the cavity of the same strength $-2 \kappa$ (defined as normalized voltage, similar to that of the deflecting cavity) is increased by a factor of $\left(\sigma_{x, T C A V 2} / \sigma_{z}\right)$. The transverse beam size is physically limited by the beam pipe radius and by the rf wavelength and can be extended into millimeter and centimeter range, while the bunch length is typically on the order of $0.1 \mathrm{~mm}$ in modern $\mathrm{x}$-ray freeelectron lasers (FELs). Summarizing the above, the TCBC scheme can be 10-100 times more efficient compared to conventional off-crest acceleration.

\section{DESIGN OF TCBC FOR MaRIE FEL}

The MaRIE x-ray FEL proposed at Los Alamos National Laboratory is currently planned to lase at the wavelength of $0.3 \AA$ [22]. The electron beam will be accelerated in a $1.3 \mathrm{GHz}$ superconducting linac reaching the energy of $12 \mathrm{GeV}$. The linac includes two bunch compression stages at $250 \mathrm{MeV}$ and $1 \mathrm{GeV}$ (referred further as BC1 and BC2, respectively). High requirements on the beam quality can be met by imposing an excessive energy chirp of $\pm 5 \mathrm{MeV}$ on the bunch in order to reduce the effect of CSR during compression in $\mathrm{BC} 2$ and preserve the required normalized emittance of $\varepsilon_{n}=0.1 \mu \mathrm{m}$. A low rf frequency and close energies of the compression stages result in a large off-crest phase of $\mathrm{rf}$ which results in the accelerating gradient about a factor of 3 smaller than the peak value. This approach is not cost efficient. Moreover, a low accelerating gradient results in the increased slice energy growth caused by the microbunching instability driven by the longitudinal space charge [23,24].

As an alternative solution, we propose to use the TCBC scheme to provide the required energy chirp. The proposed parameters of the electron beam and the TCBC components are listed in Table I. The design was developed through a compromise between the cost efficiency and simplicity of operation. The minimal TCBC cost is achieved when the 
TABLE I. TCBC parameters for the MaRIE x-ray FEL.

\begin{tabular}{lcc}
\hline \hline & BC1 & BC2 \\
\hline Electron beam & & \\
Beam energy $E=\gamma m c^{2}(\mathrm{MeV})$ & 250 & 1000 \\
Bunch charge $q(\mathrm{pC})$ & 100 & 100 \\
Bunch length $\sigma_{z}(\mu \mathrm{m})$ & 90 & 90 \\
Normalized transverse emittances $\epsilon_{n x}, \epsilon_{n y}(\mu \mathrm{m})$ & 0.1 & 0.1 \\
Normalized longitudinal emittance $\epsilon_{n z}(\mu \mathrm{m})$ & 5.72 & 5.72 \\
Uncorrelated energy spread $\gamma m c^{2} \sigma_{\Delta \gamma / \gamma, u}(\mathrm{keV})$ & 32.5 & 32.5 \\
Correlated energy spread $\gamma m c^{2} R_{65} \sigma_{z}(\mathrm{MeV})$ & 6.7 & 8.1 \\
Beam line components & & \\
Total number of cryomodules & 2 & 4 \\
Total number of 13-cell cavities & 24 & 32 \\
Cavity frequency $(\mathrm{GHz})$ & 3.9 & 3.9 \\
Effective rf length in each cavity $(\mathrm{m})$ & 0.5 & 0.5 \\
Deflecting voltage in each cavity $(\mathrm{MeV})$ & 2.5 & 2.5 \\
Number of $13-\mathrm{cell}$ cavities per TCAV & 6 & 8 \\
Drift length $D(\mathrm{~m})$ & 3.39 & 6.58 \\
TCAV length $L_{c}(\mathrm{~m})$ & 4.28 & 5.72 \\
TCAV strength $\kappa\left(\mathrm{m}{ }^{-1}\right)$ & 0.82 & 0.205 \\
$R_{65}$ (m $\left.{ }^{-1}\right)$ & -298 & -90 \\
Beam line length $(\mathrm{m})$ & 25.3 & 50.6 \\
\hline \hline
\end{tabular}

ratio of drift and TCAV lengths is roughly the same as the ratio between costs of rf infrastructure (including the cost of structures, cryomodules, tunnel, etc.) and vacuum drifts. The simplicity requirement results in a scheme which can fit within the length of the integer number of standard cryomodules, which simplifies replacement. Table I summarizes components of two different designs of the TCBC at the beam energies of $250 \mathrm{MeV}$ (right after $\mathrm{BC} 1$ ) and $1 \mathrm{GeV}$ (right before $\mathrm{BC} 2$ ). Both designs are based on the cryomodules [25] and 13-cell 3.9 GHz transverse deflecting cavities [26] designed for the International Linear Collider (ILC) [3]. The schematic layouts of TCAVs inside the cryomodules are shown in Fig. 2.

\section{A. Chirper at $250 \mathrm{MeV}$}

The required correlated energy spread of $5 \mathrm{MeV}$ for the $250 \mathrm{MeV}$ beam energy results in $\Delta \gamma / \gamma=0.02$ for particles in the head and the tail of the bunch that is well within the limits of the linear formalism (requiring $\Delta \gamma / \gamma \ll 1$ ) presented in Sec. II. Accordingly, the TCBC beam line was simulated using the particle-tracking code ELEGANT [27]

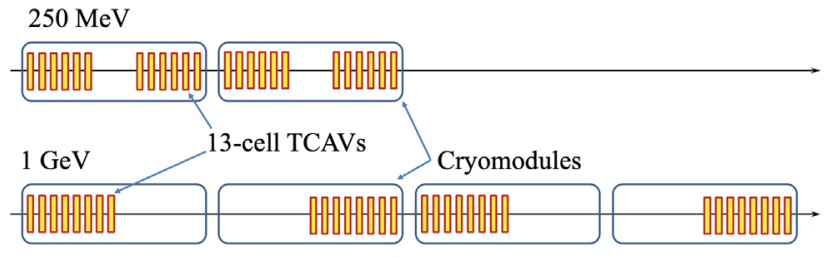

FIG. 2. The layout of TCAVs inside the cryomodules for the MaRIE linac. Two design options for TCBC at $250 \mathrm{MeV}$ (above) and $1 \mathrm{GeV}$ (below) are shown. including nonlinearities of each element up to the third order, and additionally accounting for the longitudinal space charge (LSC) for a $100 \mathrm{pC}$ bunch. The latter did not result in any visible changes in the beam dynamics in the schemes placed before $\mathrm{BC} 2$ which are discussed in this section [28]. The wakefields were not included in the simulation, since they strongly depend on the geometry of the cavity and do not represent general findings. Twiss parameters are optimized $\left(\beta_{x, y}=59 \mathrm{~m}, \alpha_{x, y}=2.1\right)$ to minimize the increase in the beam's emittance. Figure 3(a) demonstrates the evolution of the rms beam sizes along the beam line. In particular, $\sigma_{x}$ grows significantly in the middle TCAV due to introduced $z$-dependent angular spread. The transverse-to-longitudinal coupling is removed at the end of the beam line, resulting in $\sigma_{x}$ and $\sigma_{y}$ that are close to each other and slightly smaller than their initial values due to focusing in a vacuum drift for the chosen input Twiss parameters. The longitudinal beam size is almost unaffected by the transverse-to-longitudinal correlations and remains constant along the beam line. The energy chirp is defined using the conventional notations:

$$
h=\frac{\langle z \cdot \Delta \gamma / \gamma\rangle}{\left\langle z^{2}\right\rangle}\left[\mathrm{m}^{-1}\right],
$$

where $\langle\cdots\rangle$ denotes the ensemble average. It is predominantly acquired in TCAV2 [as shown in Fig. 3(b)] as was predicted by the linear formalism (see Sec. II). Figure 3(c) demonstrates evolution of the transverse $\left(\epsilon_{n x}\right.$ and $\left.\epsilon_{n y}\right)$ and longitudinal $\left(\epsilon_{n z}\right)$ normalized emittances along the beam line. The transverse and longitudinal emittances grow significantly during beam propagation, since the motions in the transverse $\left(x, x^{\prime}\right)$ and longitudinal $\left(z, z^{\prime}\right)$ phase spaces are highly coupled. The transverse emittance $\epsilon_{n x}$ is $0.21 \mu \mathrm{m}$ at the end of the beam line, which is roughly a factor of 2 larger than its initial value of $0.1 \mu \mathrm{m}$.

The emittance degradation due to nonlinear and collective effects in the schemes with transverse-to-longitudinal mixing can be investigated by using the eigenemittance analysis. Linear 6D dynamics of relativistic beams allows three invariants characterizing the electron bunch. They can be chosen to be the eigenemittances $\tilde{\lambda}_{j}$, introduced by Dragt [29,30]:

$$
\operatorname{det}\left(J \Sigma-i \tilde{\lambda}_{j} I\right)=0,
$$

where $\Sigma=\left\langle\zeta \zeta^{T}\right\rangle$ is the $6 \mathrm{D} \mathrm{rms}$ beam matrix, $\zeta=$ $\left(x, x^{\prime}, y, y^{\prime}, z, \Delta \gamma / \gamma\right)$ is the 6D coordinates of an electron in the phase space, $J$ is the unit block-diagonal antisymmetric symplectic matrix satisfying $J^{2}=-I$, and $I$ is the identity matrix. The normalized eigenemittances are defined as $\lambda_{j}=\gamma \beta \tilde{\lambda}_{j}$.

The evolution of the eigenemittances along the beam line is shown in Fig. 3(d). The transverse dynamics in $\left(y, y^{\prime}\right)$ phase space is decoupled from other dynamics and results 


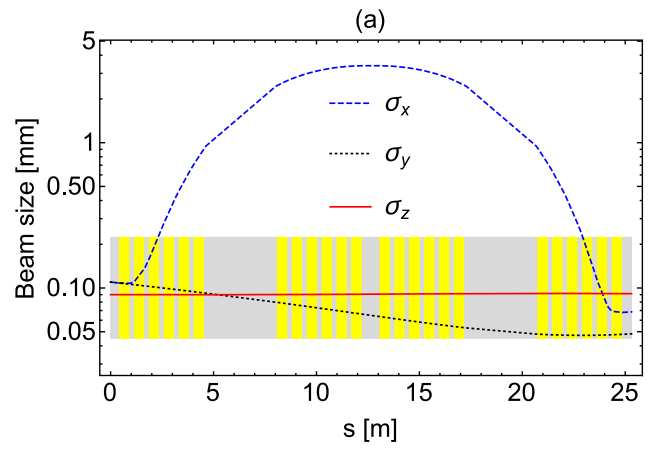

(b)

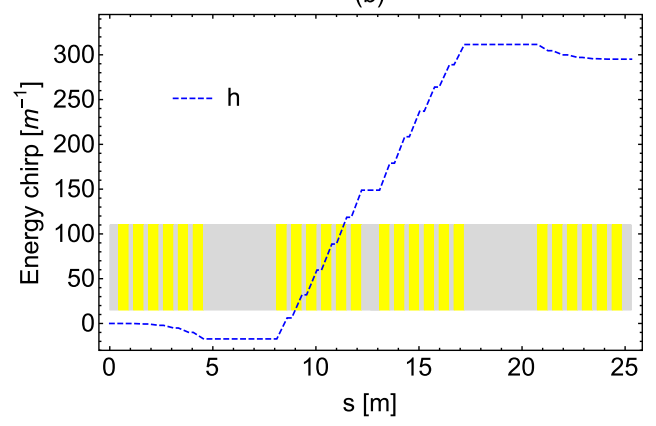

(c)

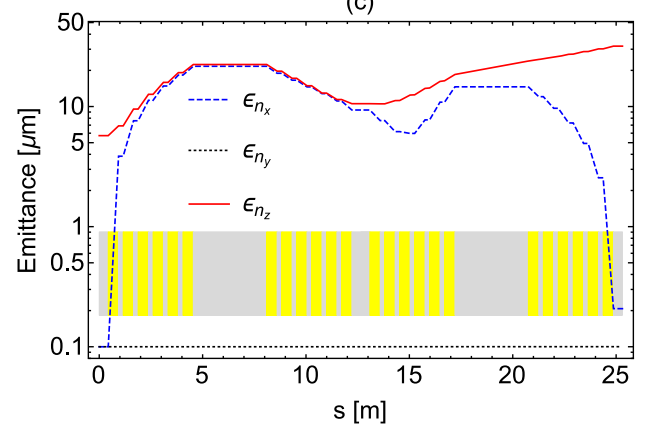

(d)

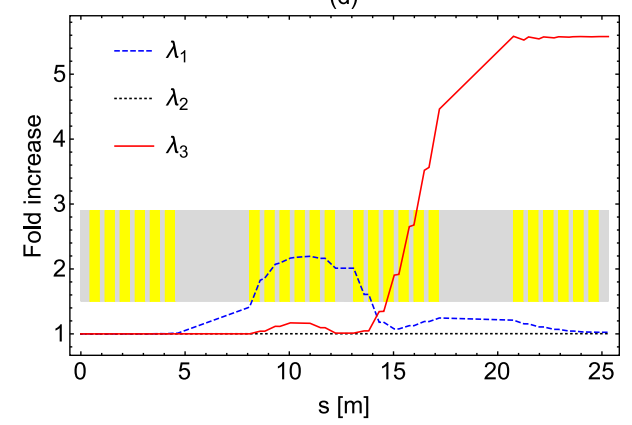

FIG. 3. The rms beam sizes (a), energy chirp (b), emittances (c), and increase in eigenemittances (d) along the beam line for the unoptimized Twiss parameters $\beta_{x}=\beta_{y}=59 \mathrm{~m}$ and $\alpha_{x}=\alpha_{y}=2.1$. Each 13-cell cavity is marked as a yellow rectangle representing its actual geometric length and position along the beam line.

in no visible changes of emittance $\epsilon_{n y}$ and the corresponding eigenemittance $\lambda_{2}$ compared to the initial value of $0.1 \mu \mathrm{m}$. The longitudinal emittance $\epsilon_{n z}=31.8 \mu \mathrm{m}$ as well as its associated eigenemittance $\lambda_{3}=31.9 \mu \mathrm{m}$ at the end of the beam line are significantly larger than their initial values of $5.72 \mu \mathrm{m}$. Longitudinal emittances and eigenemittances grow simultaneously, which indicates that their increase is caused by nonlinearities of the beam line elements, mostly due to a large imposed energy spread, $\Delta \gamma / \gamma \sim 0.2$. The eigenemittance $\lambda_{1}$ related to the transverse phase space $\left(x, x^{\prime}\right)$ at the end of the beam line predominantly grows in the middle deflecting cavity and comes back close to its original value in the last cavity, resulting in a 5\% start-toend growth. The increase in the eigenemittances in the middle of the TCBC beam line and its strong compensation at the end of the beam line is not a real physical effect. It is an artifact of the ELEGANT code, which does not use canonical variables to describe the phase space of the beam. At the same time, the significant difference between the transverse emittance $\epsilon_{n_{x}}$ and its corresponding eigenemittance $\lambda_{1}$ indicates that the phase space of the beam remains partially coupled between $\left(x, x^{\prime}\right)$ and $(z, \Delta \gamma / \gamma)$ phase planes at the end of the TCBC beam line as shown in Fig. 4. This residual coupling can be explained as follows. Nonlinear effects slightly change the average beam energy along the beam line corresponding to $\gamma_{1}=489.225$, $\gamma_{2}=489.221, \gamma_{3}=489.268$, and $\gamma_{4}=489.327$ at the entrances to TCAV1, TCAV2, TCAV3, and TCAV4, respectively. As a result, the strengths of the cavities are different from the designed values. Consequently, the effective linear matrix of the TCBC beam line has uncompensated nonzero off-diagonal elements and linear transverse-to-longitudinal correlations.

The remaining coupling between the longitudinal and transverse phase spaces can be removed through minor adjustment of the voltage in TCAV4 from 2.5 to $2.5265 \mathrm{MV}(\Delta V=+26.5 \mathrm{kV})$, while all other parameters of the beam line are unchanged. This adjustment does not significantly affect the final chirp (less then $0.12 \%$ reduction). Figure 5 illustrates that the minimum of the transverse emittance $\epsilon_{n x}$ is achieved at the same voltage when

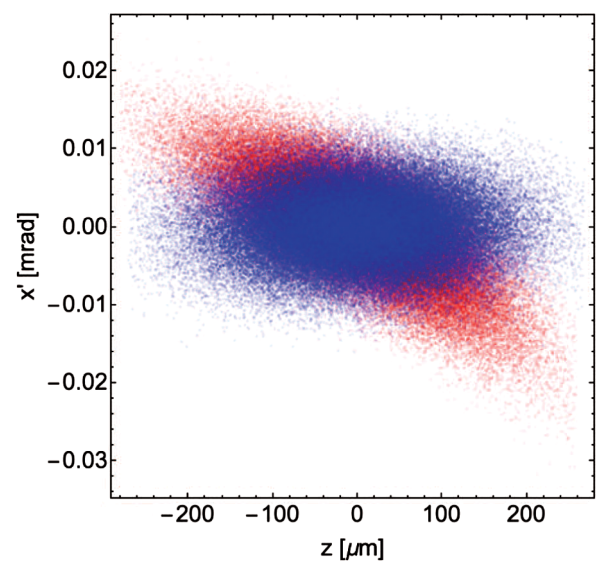

FIG. 4. Transverse phase spaces at the exit of TCBC at $250 \mathrm{MeV}$ beam energy in the linear (blue) and nonlinear regime (red) for the optimal Twiss parameters $\beta_{x, y}=59 \mathrm{~m}$ and $\alpha_{x, y}=2.1$. 


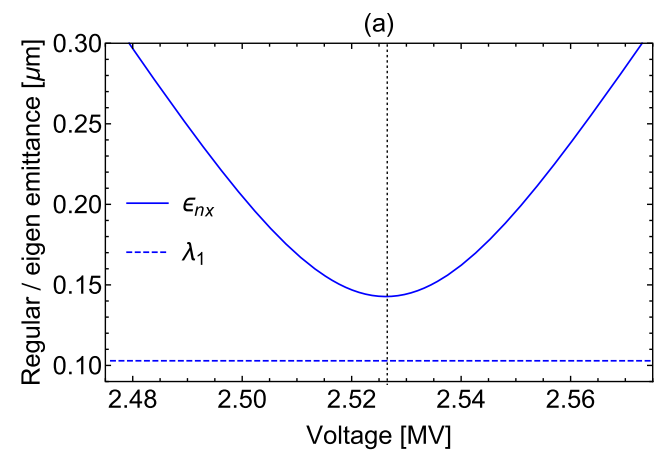

(b)

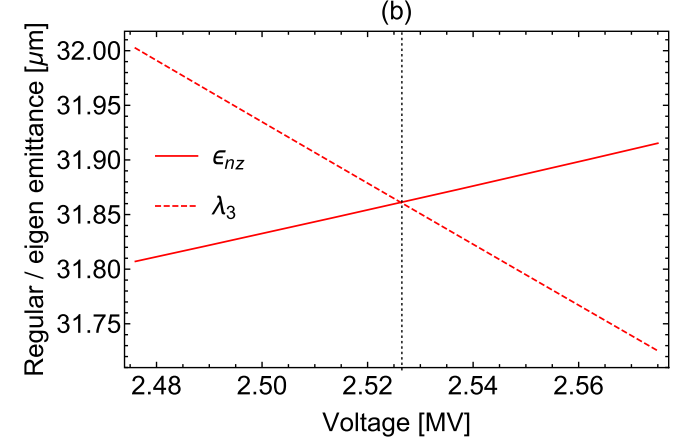

FIG. 5. (a) Transverse and (b) longitudinal emittances and corresponding eigenemittances as functions of the voltage in TCAV4. The dotted vertical line shows the optimal voltage at the minimum of the transverse emittance, which occurs when the longitudinal regular and eigenemittances match.

the longitudinal emittance matches the corresponding eigenemittances. That indicates that the longitudinal and transverse phase spaces are decoupled in this case, which is illustrated in Fig. 6.

The adjustment to the TCAV4 voltage depends nonlinearly on its design value, since it should compensate for the nonlinear effects. The TCBC scheme is independent of the sign of the deflecting voltage in TCAVs, since changing

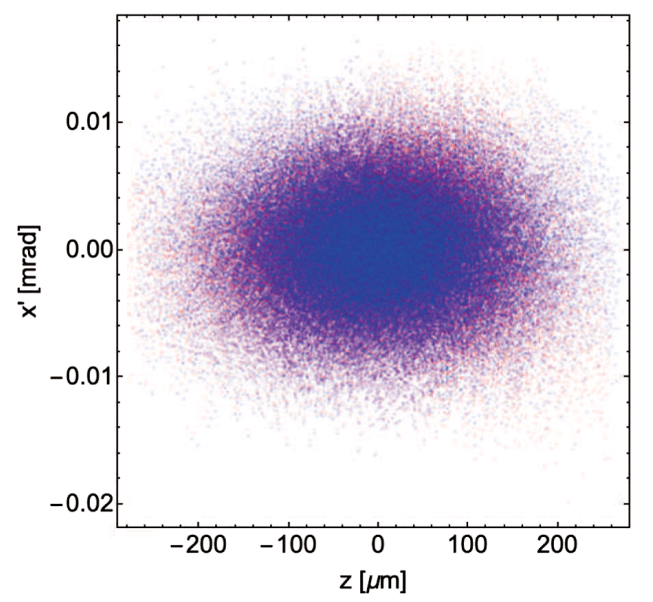

FIG. 6. Phase spaces at the exit of the TCBC at $250 \mathrm{MeV}$ with the tuned voltage $\Delta V=+26.5 \mathrm{kV}$ in the last TCAV for the linear (blue) and nonlinear (red) regimes for $\beta_{x, y}=59 \mathrm{~m}$ and $\alpha_{x, y}=2.1$. the sign is equivalent to rotating the entire beam line by $180^{\circ}$ in the $x-y$ plane along the $z$ axis. Therefore, $\operatorname{sgn}(\Delta V)=\operatorname{sgn}(V)$, which indicates that $\Delta V \propto V^{3}$. Optimization of the TCAV beam line described above resulted in the following empirical scaling for the adjusted voltage in the TCAV4:

$$
\Delta V[\mathrm{kV}] \approx 1.696 \cdot V^{3}[\mathrm{MV}]
$$

The empirically found dependence matches the theoretically expected scaling. The exact numerical factor depends on the details of the TCBC beam line, such as the beam energy, cavity length, and vacuum drift length.

Twiss parameters can be optimized to minimize the emittance growth due to the quadratic nonlinearities of the beam elements. The optimal set of $\beta_{x}=150 \mathrm{~m}$ and $\alpha_{x}=-1.3$ are significantly different from the optimized Twiss parameters prior to correction of TCAV4 voltage $\left(\beta_{x, y}=59 \mathrm{~m}\right.$ and $\left.\alpha_{x, y}=2.1\right)$, while the vertical Twiss parameters are unchanged. The transverse emittance $\epsilon_{n x}$ and the corresponding eigenemittance $\lambda_{1}$ increase by $5 \%$ for the maximum applied chirp of $300 \mathrm{~m}^{-1}$. An identical increase in these two parameters indicates that the longitudinal and transverse phase spaces are decoupled by the end of the beam line.

The output longitudinal emittance $\epsilon_{n z}$ and corresponding eigenemittance $\lambda_{3}$ are $31.86 \mu \mathrm{m}$, significantly larger than their initial values of $5.72 \mu \mathrm{m}$. The longitudinal phase space at the exit of the beam line is illustrated in Fig. 7(a). The phase space shows the residual quadratic curvature similar to the one observed during the off-crest acceleration. At the same time, the slice energy spread along the bunch is roughly the same in both linear and nonlinear analysis of the TCBC beam line as illustrated in Fig. 7(b). The curvature in the phase space can be compensated with the third harmonic cavity placed downstream in the beam line, similar to how it is done for conventional off-crest acceleration. (a)

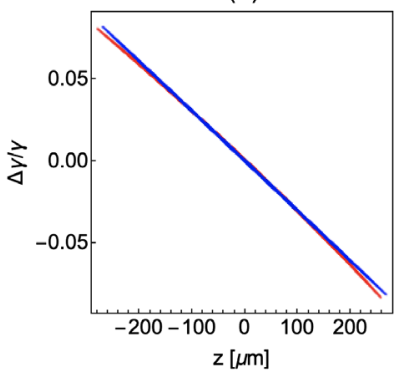

(b)

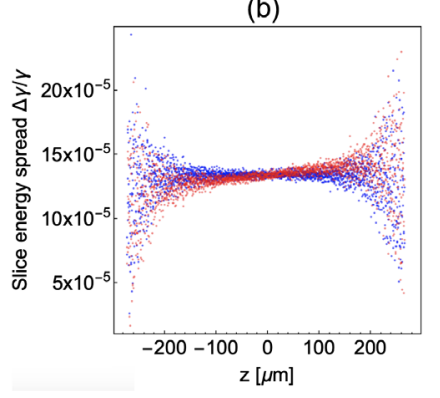

FIG. 7. Longitudinal phase space (a) and slice energy spread (b) at the exit of the TCBC at $250 \mathrm{MeV}$ with tuned voltage $\Delta V=$ $+26.5 \mathrm{kV}$ in the last TCAV and the optimized Twiss parameters of $\beta_{x, y}=150 \mathrm{~m}$ and $\alpha_{x, y}=-1.3$ in the linear (blue line) and nonlinear (red line) regimes. 


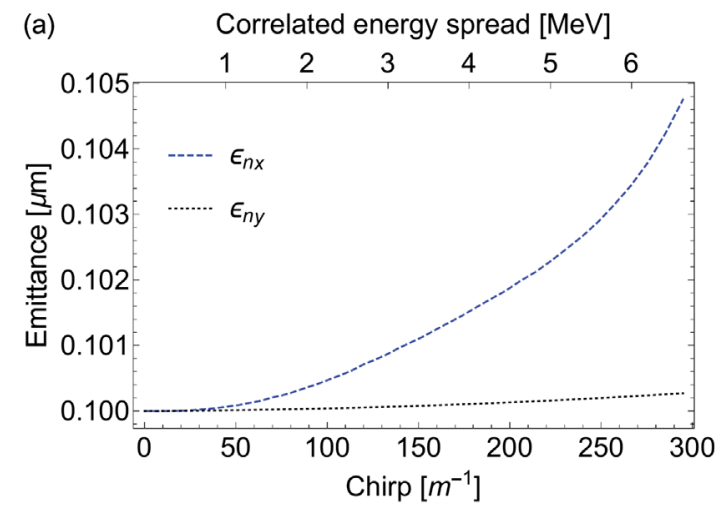

(b)

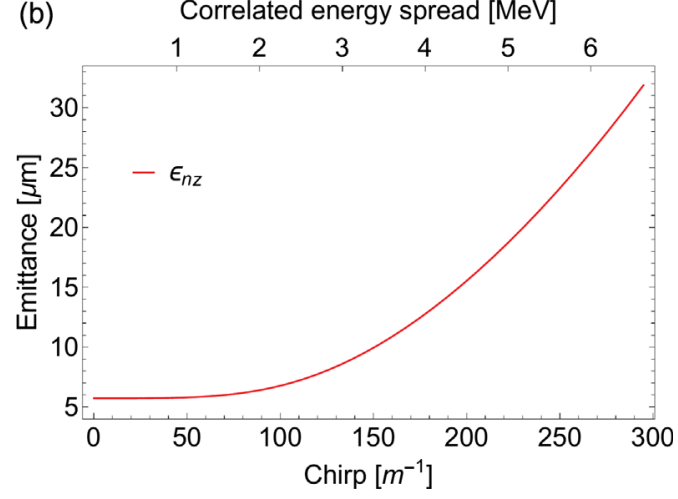

FIG. 8. (a) Transverse and (b) longitudinal emittances at the exit of the optimized TCBC at $250 \mathrm{MeV}$ versus the applied chirp.

Figure 8 summarizes the performance of the optimized TCBC beam line. The emittance degradation depends on the applied chirp, since it is caused by nonlinear effects which become more significant at larger values of imposed chirp. The imposed chirp is controlled by changing the deflecting voltage simultaneously in all cavities, while the voltage in the last cavity is adjusted according to the scaling in Eq. (7). All other parameters of the scheme are not changed, including the geometry and the Twiss parameters (Twiss parameters have been optimized for the largest imposed chirp). Optimization of Twiss parameters for each deflecting voltage does not result in significant improvement in performance.

We emphasize that optimization of the Twiss parameters and the voltage in the last cavity are equally important for the preservation of the beam quality. Optimization of the Twiss parameters without proper adjustment to the voltage in TCAV4 resulted in the smallest transverse emittance of $\epsilon_{n x}=0.2 \mu \mathrm{m}$ as shown in Fig. 3(c). On the other hand, tuning the voltage in TCAV4 without proper optimization in the Twiss parameters resulted in the smallest emittance of $\epsilon_{n x}=0.14 \mu \mathrm{m}$ as shown in Fig. 5(a). Simultaneous optimization of these parameters results in the transverse emittance of $\epsilon_{n x}=0.105 \mu \mathrm{m}$ as shown in Fig. 8(a).

The TCBC scheme employs a strong distortion of the phase space of the beam and its restoration at the end of the beam line. As a result, the scheme is vulnerable to imperfection of the beam line elements. It has been discussed above that the voltage in the TCAV4 needs to be adjusted in order to eliminate emittance growth. The numerical example presented in Fig. 5(a) shows that the transverse emittance doubles if the voltage in TCAV4 differs from the optimized value by only $1 \%$. Fortunately, the amplitude stability of modern klystrons matches this requirement [31]. Moreover, the stability requirement for the TCAV amplitude can be reduced if all the cavities are powered by a single klystron. In this case, the klystron power jitter does not affect the condition for the perfect decoupling between the longitudinal and transverse phase spaces $\kappa_{1}+\kappa_{2}+\kappa_{3}+\kappa_{4}=0$. This jitter will just result in the same jitter of the chirp $\left(R_{65} \propto \kappa^{2} \propto P_{\text {klystron }}\right)$.

The TCBC scheme is not very sensitive to the TCAV voltage jitter. At the same time, it is strongly sensitive to the rf phase jitter. The mismatch between the rf phase and the bunch arrival time results in a transverse kick to the bunch centroid [32]. That leads to the middle cavity affecting the mean energy of the bunch in addition to imposing the chirp. That, in turn, changes the strength of the TCAV4 and results in the emittance growth as shown in Fig. 5(a). We have investigated the effect of the rf phase jitter numerically. The results are shown in Fig. 9. The relative phases of the fields in TCAVs are fixed as if they are powered by a

(a)

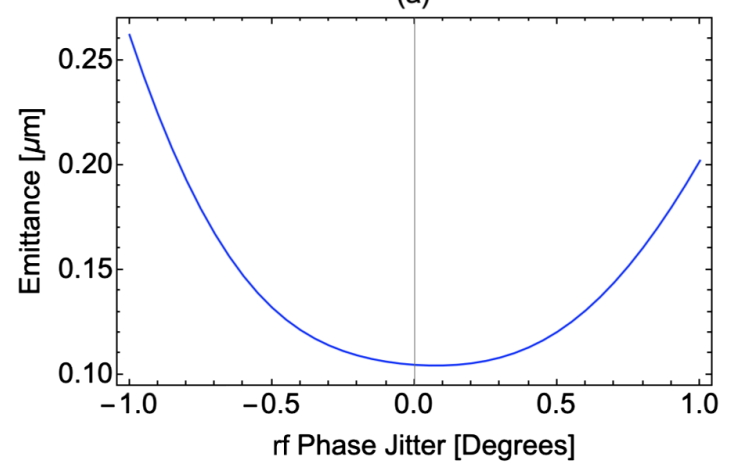

(b)

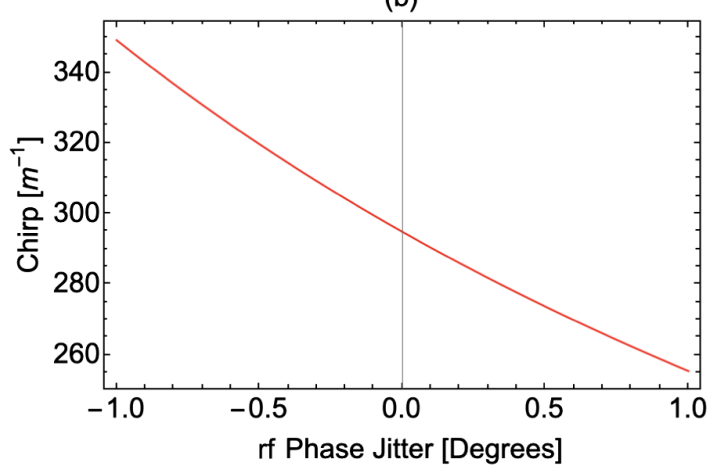

FIG. 9. Transverse emittance (a) and sensitivity of the chirp (a) to the rf phase jitter for the TCBC design at $250 \mathrm{MeV}$ and the largest anticipated imposed chirp. 
single rf source. The rf phase jitter (or bunch arrival time jitter) results in the degradation of the emittance. Typical rf jitter of $0.1^{\circ}[31]$ results in the output emittance of $0.107 \mu \mathrm{m}$.

\section{B. Chirper at $1 \mathrm{GeV}$}

The design of the TCBC at $1 \mathrm{GeV}$ fits four cryomodules, and it is significantly longer than the design of the TCBC at $250 \mathrm{MeV}$, which fits two cryomodules as illustrated in Fig. 2. It also requires more rf power, due to the larger length of the deflecting cavities assuming the same peak field in TCAVs (see Table I for more details). This difference is driven by the unfavorable scaling of the TCAV strength with energy, $\kappa \propto 1 / \gamma$, and strong dependence of the imposed chirp on the cavity's strength, $R_{65} \propto \kappa^{2}$. As a result, the imposed correlated energy spread is smaller in high-energy beams: $\sigma_{\Delta \gamma}=\gamma m c^{2} R_{65} \sigma_{z} \propto 1 / \gamma$. A longer beam line is required to impose the same correlated energy spread at higher beam energies.

At the same time, imposing the same correlated energy spread on the beam at a higher energy results in smaller relative energy spread $\Delta \gamma / \gamma$, smaller nonlinear effects, and smaller residual transverse-to-longitudinal coupling. As a result, adjustment of the voltage in TCAV4 is not required, in contrast to the TCBC at $250 \mathrm{MeV}$. The emittance growth is significantly smaller at a higher energy as shown in
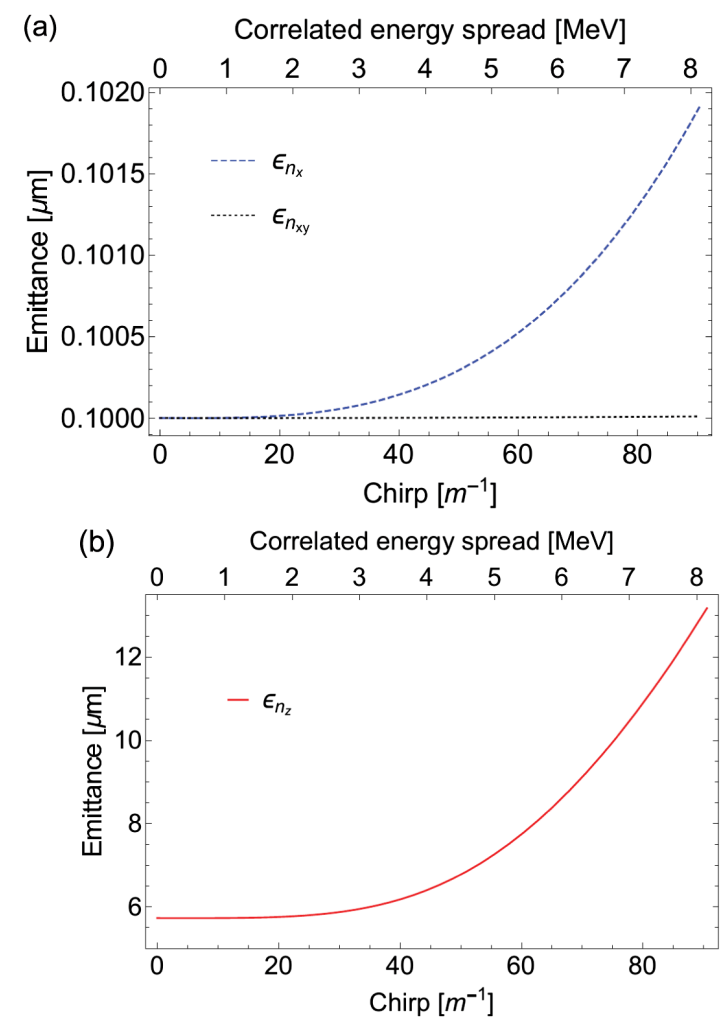

FIG. 10. (a) Transverse and (b) longitudinal emittances at the exit of the optimized TCBC at $1 \mathrm{GeV}$ versus the applied chirp.
Fig. 10. The optimized Twiss parameters in this case are $\beta_{x}=175 \mathrm{~m}$ and $\alpha_{x}=0.3$.

The designs of the TCBC at $250 \mathrm{MeV}$ (Sec. III A) and $1 \mathrm{GeV}$ (III B) present a trade-off. The TCBC is more compact and cheaper at low energy. At the same time, imposing chirp at a higher energy results in an output beam of higher quality. Moreover, the space charge effects are reduced at a higher beam energy. Imposing the chirp at a higher energy is beneficial for suppressing the microbunching instability, since the beam is accelerated faster. The choice of the energy for chirping the beam should be determined from the start-to-end simulations of the linac and thorough evaluation of the full degradation in the beam quality.

\section{TCAV-BASED DECHIRPER}

The TCAV-based chirper scheme studied in Sec. II imposes chirp equal to $R_{65}=-\frac{2}{3} \kappa^{2}\left(3 D+2 L_{c}\right)$, which quadratically depends on the cavity's strength. As a result, the sign of the chirp cannot be switched by simply changing the voltage in TCAVs to the opposite sign. Formally, the $R_{65}$ matrix element can be positive if $|D|>\frac{2}{3} L_{c}$ and $D<0$. That can be achieved through the use of a FODO lattice between the TCAVs, with the beam transform matrix identical to the drift space with a negative drift length. Details on the design of the effective negative drift can be found in the Appendix.

Inclusion of quadrupoles in the scheme preserves the concept of the transverse-to-longitudinal mixing, while the energy chirp is still predominantly changed in the middle cavity. The proposed transverse-deflecting-cavity-based dechirper (TCBD) can be used to eliminate the residual chirp after compression in the final bunch compressor of the linac. The TCBD is an active element, unlike the dechirper based on a wake excitation [33-35]. The performance of the scheme does not depend on the bunch charge, and it is insensitive to the details of the longitudinal bunch profile. The amplitude of the imposed negative chirp is controlled with rf power and can be quickly adjusted during operation.

\section{A. TCBD for MITS}

This section describes a TCAV-based dechirper design for the MaRIE injector test stand (MITS) at a beam energy

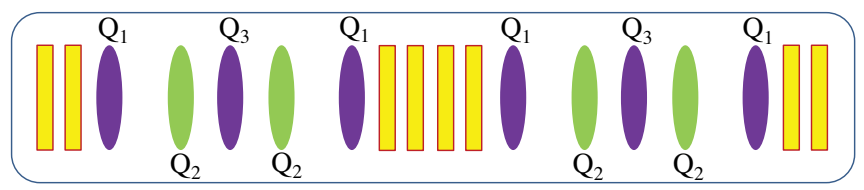

FIG. 11. The layout of the TCAV-based dechirper inside the cryomodule for MITS. Each 13-cell cavity is represented in yellow, while the focusing and defocusing quadrupoles in respect to the $\left(x, x^{\prime}\right)$ phase space are green and purple, respectively. 
TABLE II. Parameters of the TCAV-based dechirper beam line and beam for MITS.

\begin{tabular}{lcc}
\hline \hline & MITS MaRIE \\
\hline Electron beam & & \\
Beam energy $(\mathrm{MeV})$ & 250 & 1000 \\
Bunch charge $(\mathrm{pC})$ & 100 & 100 \\
Bunch length $(\mu \mathrm{m})$ & 90 & 3.9 \\
Uncorrelated energy spread $(\mathrm{keV})$ & 32.5 & 750 \\
Normalized transverse emittance $\epsilon_{n x}, \epsilon_{n y}(\mu \mathrm{m})$ & 0.1 & 0.1 \\
Normalized longitudinal emittance $\epsilon_{n z}(\mu \mathrm{m})$ & 5.72 & 5.72 \\
Uncorrelated energy spread $\gamma m c^{2} \sigma_{\Delta \gamma / \gamma, u}(\mathrm{keV})$ & 32.5 & 750 \\
Correlated energy spread $\gamma m c^{2} R_{65} \sigma_{z}(\mathrm{MeV})$ & 0.6 & 5.2 \\
Beam line components & & \\
Total number of cryomodules & 1 & 6 \\
Total number of $13-\mathrm{cell}$ cavities & 8 & 64 \\
Cavity frequency $(\mathrm{GHz})$ & 3.9 & 3.9 \\
Effective rf length in each cavity $\left(\mathrm{m}^{2}\right)$ & 0.5 & 0.5 \\
Deflecting voltage in each cavity $\left(\mathrm{MeV}^{2}\right)$ & 2.5 & 2.5 \\
TCAV length $L_{c}(\mathrm{~m})$ & 1.38 & 11.51 \\
TCAV strength $\kappa\left(\mathrm{m}{ }^{-1}\right)$ & 0.82 & 0.205 \\
Total number of quads & 10 & 10 \\
Quadrupole length $(\mathrm{m})$ & 0.05 & 0.1 \\
Quadrupole 1 geometric strength $\left(\mathrm{m}^{-2}\right)$ & 23.8 & 2.2 \\
Quadrupole 2 geometric strength $\left(\mathrm{m}^{-2}\right)$ & -28.4 & -5.0 \\
Quadrupole 3 geometric strength $\left(\mathrm{m}^{-2}\right)$ & 65.3 & 16.5 \\
Effective negative drift $(\mathrm{m})$ & -5 & -65.3 \\
$R_{65}$ (m) & 26.7 & 1333 \\
Beam line length $(\mathrm{m})$ & 13.1 & 75.9 \\
\hline \hline
\end{tabular}

of $250 \mathrm{MeV}$. The design fits inside a single standard ILC cryomodule [25]. The superconducting quadrupoles with a reasonable peak gradient are integrated in the scheme to provide the effective negative drift. The layout of the TCBD is shown in Fig. 11. The main parameters are listed in Table II. The MITS dechirper at $250 \mathrm{MeV}$ is designed to demonstrate the proof of principle of the concept rather than being a practical application. The proposed design results in $600 \mathrm{keV}$ of negative correlated energy spread, which is sufficient for an experimental demonstration.

The TCBD for MITS was analyzed in the approximation of the input beam with a zero chirp. Thus, the scheme provides a decompressing energy chirp imposed on the beam by the end of the beam line. Figure 12 shows the evolution of the main beam parameters along the beam line. Growth of the transverse emittances can be reduced by adjusting the input Twiss parameters of the beam. In contrast to the chirper beam line, the adjustment of the Twiss parameters $\beta_{x}$ and $\alpha_{x}$ should be done independently from $\beta_{y}$ and $\alpha_{y}$ for the dechirper beam line. The reason for that is the lack of symmetry in dynamics in $x$ and $y$ phase spaces. The optimization results in final transverse emittances of $\epsilon_{n_{x}}=0.1029 \mu \mathrm{m}$ and $\epsilon_{n_{y}}=0.1003 \mu \mathrm{m}$ and a final longitudinal emittance of $\epsilon_{n_{z}}=6.08 \mu \mathrm{m}$ for the optimal values of the input Twiss parameters of $\beta_{x}=28.8 \mathrm{~m}, \beta_{y}=9.8 \mathrm{~m}$, $\alpha_{x}=-7$, and $\alpha_{y}=1$. The degradation of the beam quality due to nonlinear effects is not significant at the proper choice of Twiss parameters.

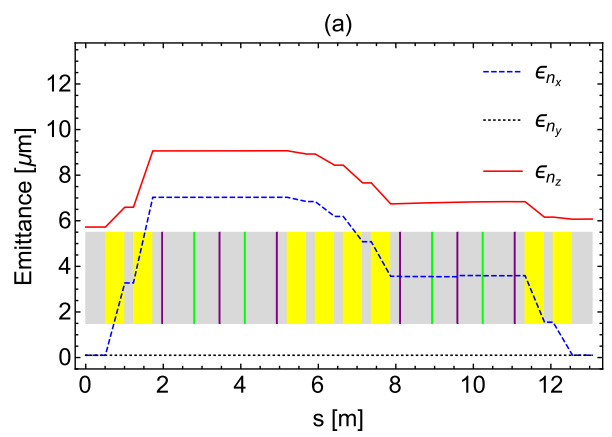

(b)

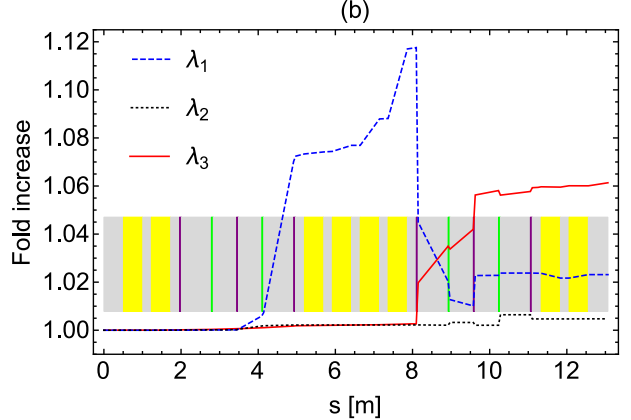

(c)

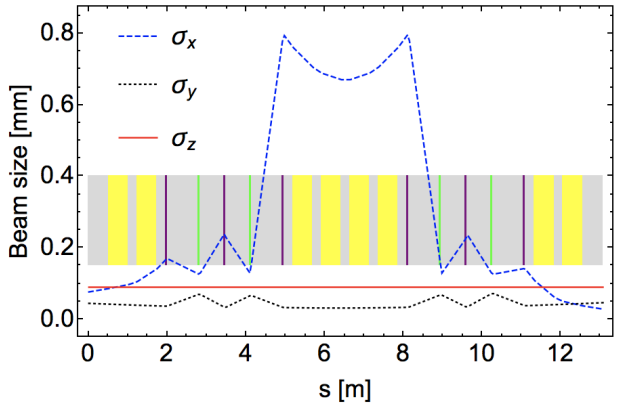

(d)

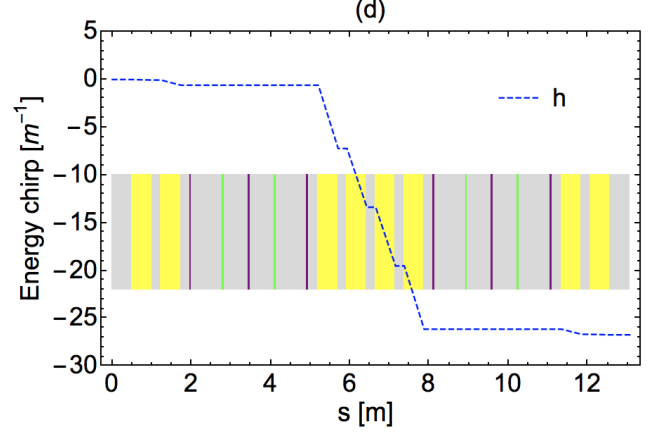

FIG. 12. Evolution of the normalized emittances (a), corresponding eigenemittances (b), rms beam sizes (c), and energy chirp (d) along the beam line of the TCAV-based dechirper at $250 \mathrm{MeV}$.

The imposed chirp in the TCBD design for MITS is about a factor of 10 smaller than in the chirper studied in Sec. III A. The nonlinear emittance growth due to the partial correlation between the longitudinal and the transverse phase spaces is not significant for these parameters. We did not adjust the deflecting voltage in the last TCAV to minimize emittance growth and did not study emittance growth versus imposed chirp in this case. 


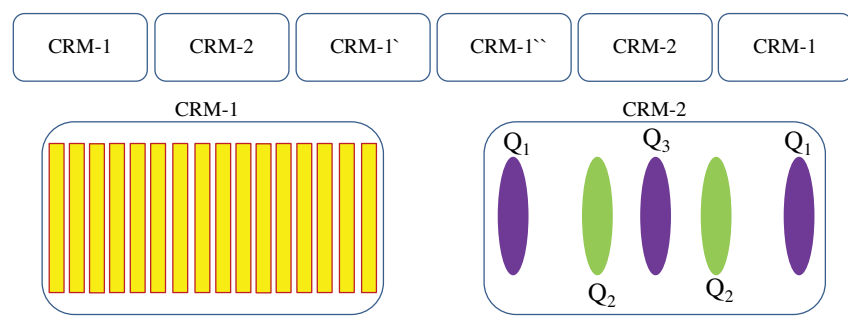

FIG. 13. The layout of the TCAV-based dechirper at $1 \mathrm{GeV}$ which fits inside six cryomodules. Cryomodules 1, 3, 4, and 6 have 16 13-cell cavities (shown in yellow). Cryomodules 2 and 5 host negative drift beam lines designed of focusing (green) and defocusing (purple) quadrupoles in respect to the $\left(x, x^{\prime}\right)$ phase space.

(a)

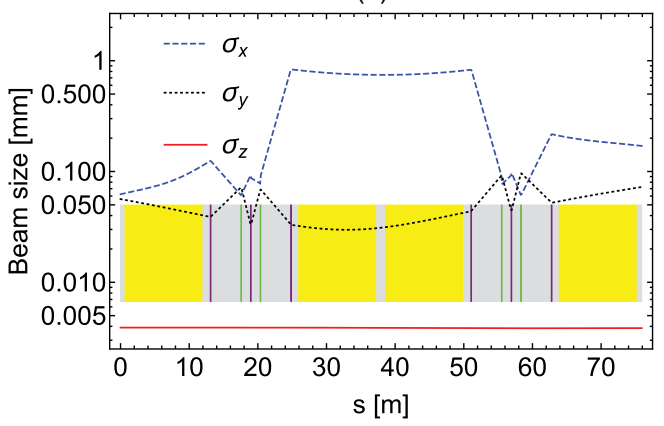

(b)

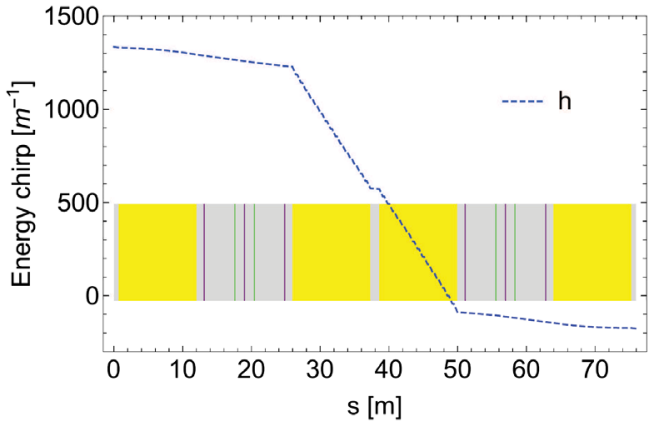

(c)

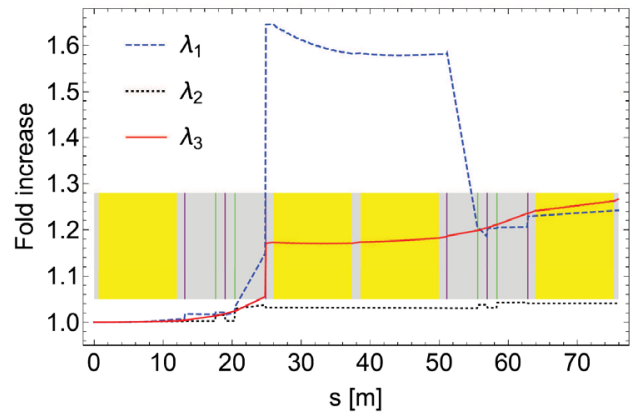

FIG. 14. The rms beam sizes (a); the total energy spread and chirp (b) and eigenemittances (c), at a distance $s$ along the beam line of the TCAV-based dechirper at $1 \mathrm{GeV}$ for Twiss parameters $\beta_{x}=76 \mathrm{~m}, \beta_{y}=62 \mathrm{~m}, \alpha_{x}=-2.5$, and $\alpha_{y}=1.6$.

\section{B. Dechirper design for the MaRIE linac at $1 \mathrm{GeV}$}

This section describes a practical design of the dechirper which may be implemented at the MaRIE linac. The overall design fits in six cryomodules as shown in Fig. 13. The length of the dechirper is significantly larger than the length of the chirper at $1 \mathrm{GeV}$ ( $\mathrm{Sec}$. III B), since the bunch is compressed down to $3.9 \mu \mathrm{m}$ compared to the precompressed value of $90 \mu \mathrm{m}$ (compression ratio of 23). The parameters of the beam line elements are listed in Table II. Identical negative drifts are implemented in the second and fifth cryomodules. Each negative drift consists of five quadrupole magnets separated by drifts. Note that the distances between quadrupoles (Fig. 13) and their strengths are different from those in a simple double triplet design described in the Appendix. The design of this negative drift represents a compromise between its efficiency and quality. It is compact enough to provide the effective negative drift of $65 \mathrm{~m}$ without much degradation in the beam quality. A chirp (up to $5.2 \mathrm{MeV}$ ) was applied to the beam at the entrance to the scheme to simulate the beam coming out of the MaRIE chicane. The TCBD scheme was simulated as an actual dechirper, and strengths of TCAVs were adjusted to eliminate the imposed chirp.

Figure 14 demonstrates the evolution of the beam parameters along the beam line. The chirp is mostly eliminated in the middle TCAV, similar to the TCBC scheme. The small residual transverse-to-longitudinal linear correlations are very small. If critical for larger normalized energy spread values, they can be adjusted by tuning the voltage in the last TCAV, similar to what was accomplished for the $250 \mathrm{MeV}$ chirper scheme. We emphasize that, unlike the chirper design, the voltage in the last cavity must be reduced compared to the linear case. The Twiss parameters are optimized to minimize emittance growth. The output transverse emittances are $\epsilon_{n x}=0.127 \mu \mathrm{m}, \epsilon_{n y}=0.104 \mu \mathrm{m}$, and $\epsilon_{n z}=7.25 \mu \mathrm{m}$ for the optimal Twiss parameters of $\beta_{x}=76 \mathrm{~m}, \beta_{y}=62 \mathrm{~m}, \alpha_{x}=-2.5$, and $\alpha_{y}=1.6$.

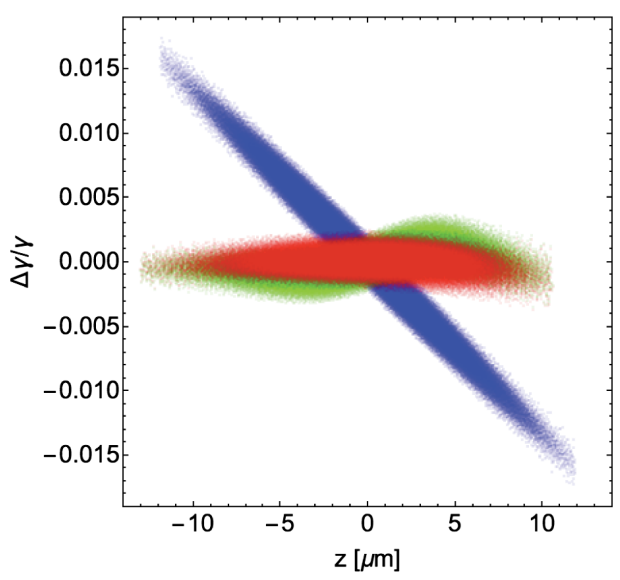

FIG. 15. Longitudinal phase space at the entrance (blue) and exit of the TCAV-based dechirper at $1 \mathrm{GeV}$ with optimized input Twiss parameters for 0 (red) and $100 \mathrm{pC}$ (green) bunches. 
The effect of the longitudinal space charge must be accounted for in the MaRIE TCBD design because of the high peak current $I=3 \mathrm{kA}$ after the beam compression and relatively low beam energy. In fact, the beam propagates in a strongly space-charge-dominated regime after compression, since $2 I / I_{A} \frac{1}{\left(\beta_{e} \gamma\right)^{3}} \sigma_{x}^{2} \gg \epsilon_{n x}^{2} / \gamma^{2}$, where $\beta_{e} \approx 1$ is the relativistic velocity and $I_{A} \approx 17 \mathrm{kA}$ is the Alfvén current. As a result, the emittances of the output beam are somewhat larger than what is expected for a bunch with zero charge $\left(\epsilon_{n x}=0.127 \mu \mathrm{m}, \epsilon_{n y}=0.101 \mu \mathrm{m}\right.$, and $\epsilon_{n z}=$ $6.54 \mu \mathrm{m})$. The transverse emittances are mostly not affected by the space charge, but the longitudinal emittance grows due to the space charge wake. Figure 15 compares the longitudinal phase space at the entrance (blue) and
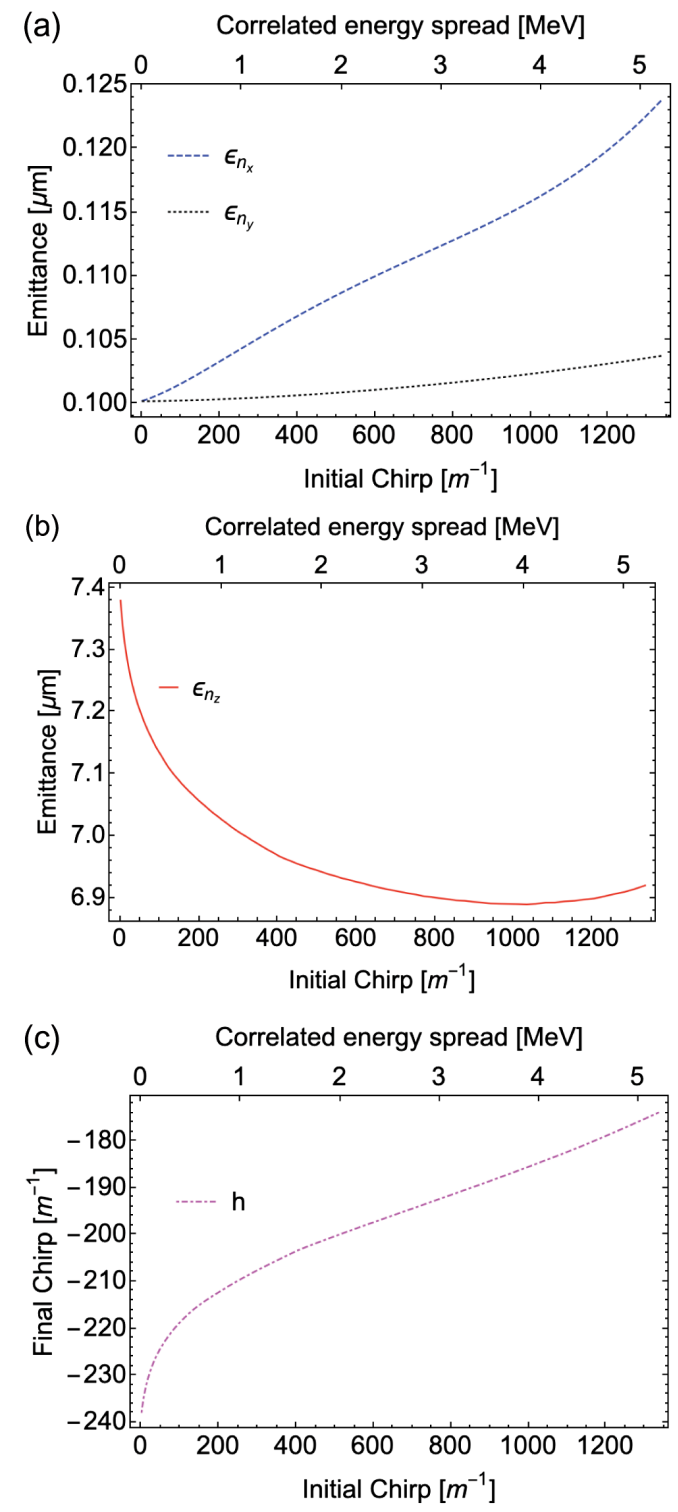

FIG. 16. Transverse (a) and longitudinal (b) emittances and chirp (c) at the exit of the optimized TCBD at $1 \mathrm{GeV}$ versus the initial chirp for a $100 \mathrm{pC}$ bunch. exit of the beam line for 0 (red) and $100 \mathrm{pC}$ (green) bunches.

Emittance growth and the final chirp are plotted versus initial chirp in Fig. 16 for a 100 pC bunch.

\section{SUMMARY}

In this paper, we have proposed and studied a novel scheme for imposing a linear energy chirp on an electron bunch using a combination of transverse deflecting cavities separated by drifts. We have designed two chirpers which can be placed at the beam energies of $250 \mathrm{MeV}$ and $1 \mathrm{GeV}$, respectively. These schemes are designed to impose the required energy chirp of $5 \mathrm{MeV}$ prior to the bunch compressor BC2. Both chirpers are efficient and cause only minor degradation to the beam quality (see Figs. 8 and 10). Compensation of the higher-order nonlinear effects is required at the low energy $(250 \mathrm{MeV})$ due to the large chirp compared to the energy of the beam. This was successfully accomplished through adjusting the voltage in the last transverse deflecting cavity and optimizing the input Twiss parameters. The design of the TCBC at a higher energy of $1 \mathrm{GeV}$ results in a better quality beam but requires a longer length of rf structures and drifts.

We have demonstrated that the TCBC concept can be modified to impose decompressing energy chirp in the beam. Such a design can be used in conventional linacs for removing residual chirp in beams after the last bunch compressor. The design requires the use of FODO lattices that effectively act as negative drifts between deflecting cavities. This approach may be superior compared to passive dechirpers based on the excitation of longitudinal wakes inside of a corrugated pipe [33-35]. The main advantage of the proposed novel scheme is full control over the beam dynamics through beam optics elements. As a result, the TCBD does not depend on the bunch charge and the details of the current distribution (although some degradation of the beam quality may be expected for high current bunches due to longitudinal space charge effects). We described the design of the TCBD at $250 \mathrm{MeV}$ for the proof-of-principle demonstration at MITS and design for the TCBD dechirper for the MaRIE linac at $1 \mathrm{GeV}$.

The TCBC and TCBD schemes are flexible and can be designed at different beam energies. This suggests that they can be employed for essentially all existing and planned accelerators where it is required to impose or remove large energy chirps. This technique is superior to the conventional off-crest acceleration, particularly in terms of $\mathrm{rf}$ power consumption. The inexpensive option of imposing strong energy chirps may reduce the size of chicanes, which will mitigate degradation of the beam quality due to CSR.

\section{ACKNOWLEDGMENTS}

One of the authors (A. M.) is grateful to Professor Philippe Piot from Northern Illinois University and Dr. Paolo Craevich from Paul Scherrer Institute for fruitful discussions. 


\section{APPENDIX: NEGATIVE DRIFT}

Negative drift $\left(R_{12} \leq 0\right.$ and $\left.R_{34} \geq 0\right)$ is a virtual element in beam optics, and it can be designed as a sequence of focusing and defocusing quadrupole magnets separated by drifts. For example, an effective negative drift along the $x$ axis can be realized through as a combination of two triplets. Each triplet consists of two focusing $(f<0)$ and one defocusing $(f>0)$ quadrupoles which are separated by identical drifts $(d=-f)$. The beam transport matrix describing a thin focusing or defocusing quadrupole acting in the $4 \mathrm{D}$ transverse phase space $\left(x, x^{\prime}, y, y^{\prime}\right)$ is

$$
M_{q_{ \pm}}(f)=\left(\begin{array}{cccc}
1 & 0 & 0 & 0 \\
\pm 1 / f & 1 & 0 & 0 \\
0 & 0 & 1 & 0 \\
0 & 0 & \mp 1 / f & 1
\end{array}\right) \text {. }
$$

The longitudinal beam dynamics is not affected by the quadrupole. The triplet matrix can be found as the product of its structural components:

$$
M_{t}(d)=M_{q_{-}}(d) \cdot M_{d}(d) \cdot M_{q_{+}}(d) \cdot M_{d}(d) \cdot M_{q_{-}}(d),
$$

which results in

$$
M_{t}(d)=\left(\begin{array}{cccc}
-1 & 3 d & 0 & 0 \\
0 & -1 & 0 & 0 \\
0 & 0 & 1 & d \\
0 & 0 & 0 & 1
\end{array}\right)
$$

The combination of two triplets results in an effective negative drift for $\left(x, x^{\prime}\right)$ phase space and a positive drift for $\left(y, y^{\prime}\right)$ phase space:

$M_{d^{-}}(d)=M_{t}(d) \cdot M_{t}(d)=\left(\begin{array}{cccc}1 & -6 d & 0 & 0 \\ 0 & 1 & 0 & 0 \\ 0 & 0 & 1 & 2 d \\ 0 & 0 & 0 & 1\end{array}\right)$.

In general, the neighboring focusing elements of two triplets can be combined as a single quadrupole with a twice shorter focal length.

[1] S. Kurokawa and E. Kikutani, Overview of the KEKB accelerators, Nucl. Instrum. Methods Phys. Res., Sect. A 499, 1 (2003).

[2] C. W. Leemann, D. R. Douglas, and G. A. Krafft, The continuous electron beam accelerator facility: CEBAF at the Jefferson Laboratory, Annu. Rev. Nucl. Part. Sci. 51, 413 (2001).
[3] H. Baer, T. Barklow, K. Fujii, Y. Gao, A. Hoang, S. Kanemura, J. List, H. E. Logan, A. Nomerotski, M. Perelstein et al., The International Linear Collider technical design report-Volume 2: Physics, arXiv:1306.6352.

[4] http://aps.anl.gov/.

[5] http://bnl.gov/ps.

[6] W. Ackermann, G. Asova, V. Ayvazyan, A. Azima, N. Baboi, J. Bhr, V. Balandin, B. Beutner, A. Brandt, A. Bolzmann et al., Operation of a free-electron laser from the extreme ultraviolet to the water window, Nat. Photonics 1, 336 (2007).

[7] E. Allaria, R. Appio, L. Badano, W. Barletta, S. Bassanese, S. Biedron, A. Borga, E. Busetto, D. Castronovo, P. Cinquegran et al., Highly coherent and stable pulses from the FERMI seeded free-electron laser in the extreme ultraviolet, Nat. Photonics 6, 699 (2012).

[8] P. Emma, R. Akre, J. Arthur, R. Bionta, C. Bostedt, J. Bozek, A. Brachmann, P. Bucksbaum, R. Coffee, F.-J. Decker et al., First lasing and operation of an ångstrom-wavelength freeelectron laser, Nat. Photonics 4, 641 (2010).

[9] T. Ishikawa, H. Aoyagi, T. Asaka, Y. Asano, N. Azumi, T. Bizen, H. Ego, K. Fukami, T. Fukui, Y. Furukawa et al., A compact X-ray free-electron laser emitting in the subångström region, Nat. Photonics 6, 540 (2012).

[10] T. Tschentscher, C. Bressler, J. Grnert, A. Madsen, A. Mancuso, M. Meyer, A. Scherz, H. Sinn, and U. Zastrau, Photon beam transport and scientific instruments at the European XFEL, Appl. Sci. 7, 592 (2017).

[11] I Ko, H.-S. Kang, H. Heo, C. Kim, G. Kim, C.-K. Min, H. Yang, S. Baek, H.-J. Choi, G. Mun et al., Construction and commissioning of PAL-XFEL facility, Appl. Sci. 7, 479 (2017).

[12] C. J. Milne, T. Schietinger, M. Aiba, A. Alarcon, J. Alex, A. Anghel, V. Arsov, C. Beard, P. Beaud, S. Bettoni et al., SwissFEL: The Swiss X-ray Free Electron Laser, Appl. Sci. 7, 720 (2017).

[13] L. V. Iogansen and M. S. Rabinovich, Coherent electron radiation in the synchrotron. II, Zh. Eksp. Teor. Fiz. 37, 118 (1959) [Sov. Phys. JETP 37, 83 (1960)].

[14] B. E. Carlsten and T. O. Raubenheimer, Emittance growth of bunched beams in bends, Phys. Rev. E 51, 1453 (1995).

[15] E. L. Saldin, E. A. Schneidmiller, and M. V. Yurkov, On the coherent radiation of an electron bunch moving in an arc of a circle, Nucl. Instrum. Methods Phys. Res., Sect. A 398, 373 (1997).

[16] R. Li, Curvature-induced bunch self-interaction for an energy-chirped bunch in magnetic bends, Phys. Rev. Accel. Beams 11, 024401 (2008).

[17] J. Arthur, P. Anfinrud, P. Audebert, K. Bane, I. Ben-Zvi, V. Bharadwaj, R. Bionta, P. Bolton, M. Borland, P. H. Bucksbaum et al., Linac Coherent Light Source (LCLS) conceptual design report, SLAC National Laboratory Report No. SLAC-R-593, 2002.

[18] J. Lewellen, B. Carlsten, F. Krawczyk, Q. Marksteiner, R. Sheffield, and N. Yampolsky, MaRIE X-FEL linac design: Status and plans, Los Alamos National Laboratory Report No. LA-UR-15-21962, 2015.

[19] Y. Ding, C. Behrens, P. Emma, J. Frisch, Z. Huang, H. Loos, P. Krejcik, and M.-H. Wang, Femtosecond X-ray 
pulse temporal characterization in free-electron lasers using a transverse deflector, Phys. Rev. Accel. Beams 14, 120701 (2011).

[20] The restriction of having the same rf amplitude in all the cavities is redundant. It is sufficient that the side cavities have the same strength and the middle TCAV is twice stronger than the side ones. We assume the same ratios in their lengths for simplicity of the final expressions.

[21] M. Cornacchia and P. Emma, Transverse to longitudinal emittance exchange, Phys. Rev. Accel. Beams 5, 084001 (2002).

[22] http://marie.lanl.gov/.

[23] E. L. Saldin, E. A. Schneidmiller, and M. V. Yurkov, Klystron instability of a relativistic electron beam in a bunch compressor, Nucl. Instrum. Methods Phys. Res., Sect. A 490, 1 (2002).

[24] Z. Huang, M. Borland, P. Emma, J. Wu, C. Limborg, G. Stupakov, and J. Welch, Suppression of microbunching instability in the linac coherent light source, Phys. Rev. Accel. Beams 7, 074401 (2004).

[25] T. J. Peterson, M. Geynisman, A. Klebaner, V. Parma, L. Tavian, and J. Theilacker, in ILC cryogenic systems reference design, AIP Conf. Proc. 985, 1565 (2018).

[26] M. McAshan and R. Wanzenberg, RF design of a transverse mode cavity for kaon separation, Fermi National Accelerator Laboratory Report No. FERMILAB-TM2144, 2001.

[27] M. Borland, Elegant: A flexible SDDS-compliant code for accelerator simulation, Advanced Photon Source Report No. LS-287, 2000.
[28] LSC effects' impact on the beam dynamics in the schemes located before $\mathrm{BC} 2$ becomes visible at $10 \mathrm{nC}$ bunch charge.

[29] A. J. Dragt, F. Neri, and G. Rangarajan, General moment invariants for linear Hamiltonian systems, Phys. Rev. A 45, 2572 (1992).

[30] A. J. Dragt, Lie methods for nonlinear dynamics with applications to accelerator physics, http://www.physics .umd.edu/dsat/dsatliemethods.html, 2001.

[31] H.-S Kang, H. Yang, G. Kim, H. Heo, I. Nam, C.-K. Min, C. Kim, S. Y. Baek, H.-J. Choi, G. Mun et al., FEL performance achieved at PAL-XFEL using a three-chicane bunch compression scheme, J. Synchrotron Radiat. 26, 1127 (2019).

[32] The energy jitter resulting from the time jitter (or rf phase jitter) can be estimated from the linear beam matrix transform described with Eq. (4) as $\delta E=c R_{65} \delta t$, $\delta E=-c /(2 \pi f) R_{65} \delta \phi$.

[33] K. L. F. Bane and G. Stupakov, Corrugated pipe as a beam dechirper, Nucl. Instrum. Methods Phys. Res., Sect. A 690 , 106 (2012).

[34] P. Emma, M. Venturini, K. L. F. Bane, G. Stupakov, H.-S. Kang, M.S. Chae, J. Hong, C.-K. Min, H. Yang, T. Ha et al., Experimental Demonstration of Energy-Chirp Control in Relativistic Electron Bunches Using a Corrugated Pipe, Phys. Rev. Lett. 112, 034801 (2014).

[35] S. Antipov, S. Baturin, C. Jing, M. Fedurin, A. Kanareykin, C. Swinson, P. Schoessow, W. Gai, and A. Zholents, Experimental Demonstration of Energy-Chirp Compensation by a Tunable Dielectric-Based Structure, Phys. Rev. Lett. 112, 114801 (2014). 